\title{
Scrub typhus, a reemerging zoonosis - An Indian case series
}

\author{
Deepak Madi', Basavaprabhu Achappa ${ }^{2}$, M. Chakrapaniं ${ }^{3}$, M. R. Pavan ${ }^{4}$, Saritha Narayanan ${ }^{5}$, \\ Sujitha Yadlapati ${ }^{6}$, Sujani Yadlapati ${ }^{7}$, Soundarya Mahalingam ${ }^{8}$
}

${ }^{1}$ Associate Professor, Department of Internal Medicine, Kasturba Medical College, Mangalore (affiliated to Manipal University), ${ }^{2}$ Associate Professor, Department of Internal Medicine, Kasturba Medical College, Mangalore (affiliated to Manipal University), ${ }^{3}$ Professor, Department of Internal Medicine, Kasturba Medical College, Mangalore (affiliated to Manipal University), ${ }^{4}$ Associate Professor, Department of Internal Medicine, Kasturba Medical College, Mangalore (affiliated to Manipal University), ${ }^{5}$ Post Graduate, Department of Internal Medicine, Kasturba Medical College, Mangalore (affiliated to Manipal University), ${ }^{6}$ Intern, Department of Internal Medicine, Kasturba Medical College, Mangalore (affiliated to Manipal University), ${ }^{7}$ Intern, Department of Internal Medicine, Kasturba Medical College, Mangalore (affiliated to Manipal University), ${ }^{8}$ Associate Professor, Department of Paediatrics, Kasturba Medical College, Mangalore (affiliated to Manipal University)

\section{A B S T R A C T}

Background: Rickettsial diseases are reemerging in many parts of our country. Scrub typhus is a rickettsial disease caused by Orientia tsutsugamushi. It is difficult to diagnose Scrub typhus because of nonspecific clinical presentation and lack of availability of specific tests (ELISA) in all centres. Aim: Our aim was to study the clinical features, lab parameters and outcome of patients diagnosed with Scrub typhus. Materials and Methods: A retrospective hospital based study was done in Mangalore (Karnataka) to identify cases of scrub typhus. Patients who had an acute febrile illness and IgM antibodies against $O$. tsutsugamushi were included in our study. Results: 10 cases of Scrub typhus were identified. Among them $7(70 \%)$ patients were females and $3(30 \%)$ were males. Major symptoms on admission were fever $10(100 \%)$, dyspnea or cough-2(20\%), rashes $1(10 \%)$, altered sensorium $1(10 \%)$. The major signs were fever $10(100 \%)$, eschar $2(20 \%)$, and signs of meningial irritation in $1(10 \%)$. Lab parameters showed leukocytosis in $4(40 \%)$, thrombocytopenia in $3(30 \%)$, raised liver enzymes in $3(30 \%)$ and renal failure in $3(30 \%)$. Chest X-ray showed consolidation in $2(20 \%)$ patients. There was no mortality in our study. Conclusion: Scrub typhus can present with varying clinical manifestations and eschar can be absent in majority of cases as shown in our study. Therefore diagnosis should be based on a high index of suspicion and empirical treatment with doxycycline must be started whenever there is a strong suspicion of Scrub typhus.

Key words: Rickettsial disease, Scrub typhus, Eschar, Orientia tsutsugamushi

\section{INTRODUCTION}

Malaria, Leptospirosis, Typhoid fever, viral hepatitis and Dengue are common causes of fever in the tropics. Rickettsial disease can also present as pyrexia of unknown origin in the tropics. Rickettsial diseases once thought to have been eradicated from India are reemerging in many parts of our country. ${ }^{1,2}$

Scrub typhus is a rickettsial disease caused by Orientia tsutsugamushi. Scrub typhus is rarely diagnosed in our country because of its nonspecific clinical presentation, low index of suspicion and lack of adequate diagnostic facilities. ${ }^{3}$ The main aim of our study was to analyze the clinical features, lab parameters and outcome of patients diagnosed with Scrub typhus in our hospital.

\section{METHODOLOGY}

This retrospective study was conducted in the hospitals attached to Kasturba Medical College, Mangalore a tertiary care institution in South India. Patients admitted to our 
hospital during the period of August 2012 to August 2013 were included in this study.

Patients with an acute febrile illness who had IgM antibodies against $O$. tsutsugamushi(Scrub Typhus Detect ${ }^{\mathrm{TM}}$ IgMELISA, InBios, USA)were included in our study. ${ }^{4}$ Medical records of patients were reviewed. The clinical manifestations, investigations and outcomes were analyzed.

In our study we defined leukocytosis as white blood cells $\left[\right.$ WBC] $>12000$ cells $/ \mathrm{mm}^{3}$, anemia as hemoglobin $<10 \mathrm{~g} / \mathrm{dL}$, thrombocytopenia as platelets $<1.5 \mathrm{lakhs} / \mathrm{mm}^{3}$, elevated bilirubin as total bilirubin $>3 \mathrm{mg} / \mathrm{dL}$, elevated aspartate aminotransferase and alanine aminotransferase as four times the normal and acute renal failure as creatinine $>1.5 \mathrm{mg} / \mathrm{dL} .{ }^{5}$ This study was done after obtaining the approval from the institutional ethics committee.

\section{RESULT}

We studied 10 patients, who had been diagnosed to have Scrub typhus. Of those $7(70 \%)$ patients were females and $3(30 \%)$ were males. $3(30 \%)$ had diabetes and $2(20 \%)$ had hypertension.

Major symptoms on admission were fever-10(100\%), gastrointestinal disturbances $2(20 \%)$, dyspnea or cough $2(20 \%)$, rashes $1(10 \%)$ and altered sensorium $1(10 \%)$.

The major signs were fever-10(100\%), hepatomegaly $4(40 \%)$, splenomegaly $2(20 \%)$, eschar $2(20 \%)$, icterus $1(10 \%)$ and signs of meningial irritation $1(10 \%)$ (Table 1$)$.

Lab parameters showed leukocytosis in 4(40\%), thrombocytopenia in $3(30 \%)$, raised liver enzymes in $3(30 \%)$ and renal failure in $3(30 \%)$. Chest X-ray showed consolidation in $2(20 \%)$ patients. CSF analysis of 1 patient with features of meningitis showed total cell count 60 , of which $94 \%$ were lymphocytes. CSF protein $83.5 \mathrm{mg} / \mathrm{dl}$ and CSF glucose $59 \mathrm{mg} / \mathrm{dl}$.

All 10 patients became afebrile after initiation of Doxycycline. Their fever touched baseline within 1-3 days of Doxycycline. There was no mortality in our study. One patient who recovered from meningo-encephalitis was bedridden at the time of discharge. 8 cases were empirically treated with broad spectrum antibiotics before doxycycline was started.

\section{DISCUSSION}

Rickettsial diseases in India have been documented in Himachal Pradesh, Rajasthan, Assam, West Bengal,
Pondicherry, Kerala and Tamilnadu. ${ }^{6}$ In last few years there have been outbreaks of scrub typhus in states of south India especially Karnataka. ${ }^{5}$

Scrub typhus is transmitted to humans by the bite of larval trombiculid mites. These larval mites usually feed on the wild rats. Man is accidentally infected when he visits mite infested areas.

The clinical manifestations of this disease vary from subclinical infection to a fatal systemic illness. Patients from South India predominantly have four basic, but overlapping, presentations: mild disease (mild elevation of hepatic transaminases, leukocytosis and thrombocytopenia) with or without a rash, respiratory disease, meningoencephalitis and sepsis syndrome. ${ }^{7}$ We saw all four types of presentation in our study.

A necrotic eschar at the inoculating site of the mite is pathognomic of scrub typhus. Eschars do not cause pain or itching. The characteristic eschar and rash need not be always present. Eschar was evident in $45.5 \%$ patients in a study done by Chrispal et al. ${ }^{7}$ In our study only $2(20 \%)$ patients had eschar (Figure 1). There is a significant difference in the distribution of eschars between males and females. ${ }^{8}$ The occurrence of eschar is less frequent among South-East Asian patients. ${ }^{5}$ It has been reported that in dark-skinned patients early eschar could be easily overlooked and the eschar may be very small. ${ }^{9}$ It is important to carefully examine the front chest, areas where the skin is folded and areas within $30 \mathrm{~cm}$ below the umbilicus in both sexes, lower limbs of males and the front chest and back in females for Eschars. ${ }^{10}$ Skin rash is seen in $53 \%$ of cases. ${ }^{11}$

Serological tests remain an indispensable tool in the diagnosis of Scrub typhus. Serological tests for Rickettsial

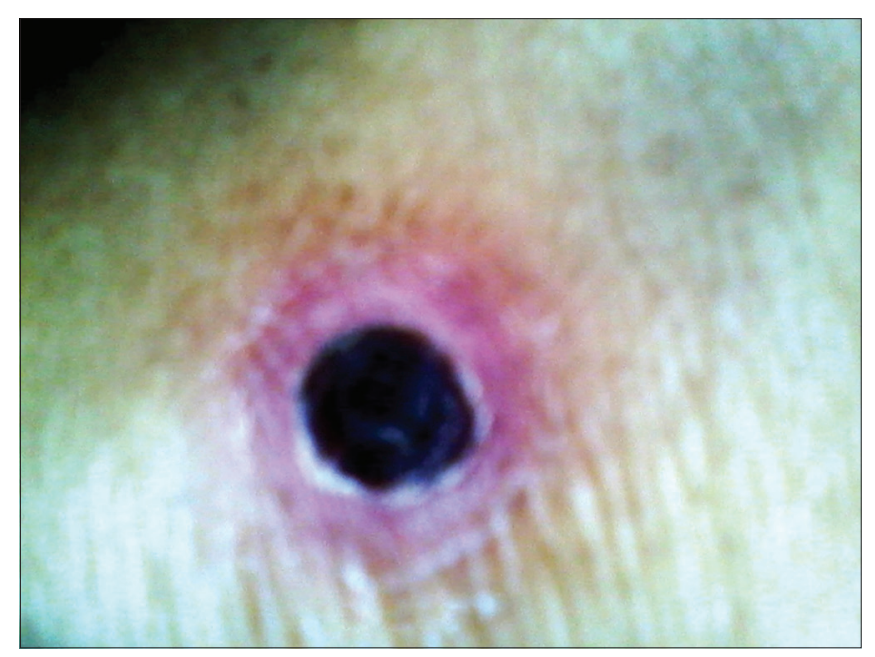

Figure 1: Eschar 


\begin{tabular}{|c|c|c|c|c|c|c|c|}
\hline SI no. & Age (years) & Gender & Symptoms & Other system involvement & Eschar & IgM positive & Outcome \\
\hline 1 & 51 & $\mathrm{M}$ & Fever, dyspnoea & $\begin{array}{l}\text { Pneumonia, hepatomegaly, } \\
\text { icterus, renal failure }\end{array}$ & No & Yes & Improved \\
\hline 2 & 54 & M & Fever, rashes & Splenomegaly & No & Yes & Improved \\
\hline 3 & 51 & $\mathrm{~F}$ & Fever & Nil & No & Yes & Improved \\
\hline 4 & 64 & $\mathrm{~F}$ & Fever, pain abdomen, diarrhoea & Hepatomegaly & Yes & Yes & Improved \\
\hline 5 & 61 & M & Fever & Splenomegaly & No & Yes & Improved \\
\hline 6 & 46 & $\mathrm{~F}$ & Fever, cough & Pneumonia & No & Yes & Improved \\
\hline 7 & 43 & $\mathrm{~F}$ & Fever & Hepatomegaly & No & Yes & Improved \\
\hline 8 & 26 & $\mathrm{~F}$ & Fever, diarrhoea & Hepatomegaly & No & Yes & Improved \\
\hline 9 & 67 & $\mathrm{~F}$ & Fever, altered sensorium & Meningitis, renal failure & No & Yes & Improved \\
\hline 10 & 71 & $\mathrm{~F}$ & Fever & Nil & Yes & Yes & Improved \\
\hline
\end{tabular}

diseases including the specific $\operatorname{IgM}$ antibody tests become positive only in the second week. ${ }^{12}$ Indirect immunofluorescence assay (IFA) is considered the test of choice. ${ }^{13}$ ELISA and Polymerase chain reaction (PCR) are other tests that can be used to diagnose scrub typhus. The Weil-Felix test using the Proteus OXK strain is in use for many years. A minimum positive titer is 1:80 or a fourfold rise over previous levels is significant. ${ }^{14}$ Patients who had IgM antibodies against $O$. tsutsugamushi were included in our study.

Penicillins and cephalosporins which are commonly used to treat pyrexia of unknown origin are inactive against rickettsiae. Doxycycline, $200 \mathrm{mg} / \mathrm{d}$ orally is the drug of choice. If a patient fails to respond to treatment with a tetracycline within 48 hours the diagnosis of rickettsial disease must be reconsidered. ${ }^{12}$. All our patients responded to doxycycline. Age $\geq 60$ years, absence of eschar, WBC counts $>10,000 / \mathrm{mm}^{3}$ and albumin $\leq 3.0 \mathrm{~g} / \mathrm{dL}$ predict the occurrence of severe scrub typhus. ${ }^{15}$ Patients withscrub typhus who have low body temperature, crepitations and elevated aspartate aminotransferase may also develop severe disease. ${ }^{16}$

The main strength of our study is that we included patients who had IgM antibodies against $O$. tsutsugamushi. Patients who had Weil-Felix test positive without IgM antibodies were excluded from the study. A small sample size is another limitation of our study but even in this small sample we came across varying presentations of this disease.

Diagnosis of Scrub typhus is difficult in our country because of its varying clinical presentation, absence of eschar in majority of cases, lack of availability of specific tests (ELISA) in all centres and the fact that serological tests become positive only in the second week. Delay in treatment can lead to complications and fatal outcome in Scrub typhus. Therefore diagnosis should be largely based on a high index of suspicion and empirical treatment with doxycycline must be started whenever there is a strong suspicion of Scrub typhus. A detailed physical examination especially over the covered areas must be performed in patients with fever to detect eschar. If clinicians do not consider a possibility of Rickettsial diseases in a patient with fever, PUO work up may become expensive.

\section{REFERENCES}

1. Mahajan SK. Rickettsial diseases. J Assoc Physicians India 2012; 60:37-44.

2. Stephen S, Kandhakumari G, Pradeep J, Vinithra SM, Siva PK, Hanifah M, et al. Scrub typhus in South India: a re-emerging infectious disease. Jpn J Infect Dis 2013;66(6):552-554.

3. Saifudheen K, Kumar KG, Jose J, Veena V and Gafoor VA. First case of scrub typhus with meningoencephalitis from Kerala: An emerging infectious threat. Ann Indian Acad Neurol 2012; $15: 141-144$

4. Viswanathan S, Muthu V, Iqbal N, Remalayam B and George T. Scrub Typhus Meningitis in South India - A Retrospective Study. PLoS One 2013; 8(6):e66595.

5. Razak A, Sathyanarayanan V, Prabhu M, Sangar $M$ and Balasubramanian R. Scrub typhus in Southern India: are we doing enough? Trop Doct 2010;40:149-151.

6. Mahajan SK, Kashyap R, Kanga A, Sharma V, Prasher BS and Pal LS. Relevance of Weil-Felix test in diagnosis of scrub typhus in India. J Assoc Physicians India 2006; 54:619-621.

7. Chrispal A, Boorugu H, Gopinath KG, Prakash JA, Chandy S, Abraham OC, et al. Scrub typhus: an unrecognized threat in South India - clinical profile and predictors of mortality. Trop Doct $2010 ; 40: 129-133$.

8. Kundavaram AP, Jonathan AJ, Nathaniel SD and Varghese GM. Eschar in scrub typhus:a valuable clue to the diagnosis. J Postgrad Med 2013;59(3):177-178.

9. Premaratna R, Chandrasena TG, Dassayake AS, Loftis $A D$, Dasch GA and de Silva HJ. Acute hearing loss due to scrub typhus: a forgotten complication of a reemerging disease. Clin Infect Dis 2006; 1:42:e6-e8.

10. Kim DM, Won KJ, Park CY, Yu KD, Kim HS, Yang TY, et al. Distribution of eschars on the body of scrub typhus patients: a prospective study. Am J Trop Med Hyg 2007; 76 (5): 806809.

11. Lee JH, Lee JH, Chung KM, Kim ES, Kwak YG, Moon C, et al. Dynamics of clinical symptoms in patients with scrub typhus. Jpn $\mathrm{J}$ Infect Dis 2013; 66(2):155-157.

12. Vivekanandan M, Mani A, Priya YS, Singh AP, Jayakumar S 
and Purty S. Outbreak of scrub typhus in Pondicherry. J Assoc Physicians India 2010; 58:24-28.

13. Chaudhry D, Garg A, Singh I, Tandon C and Saini R. Rickettsial diseases in Haryana: Not an uncommon entity. J Assoc Physicians India 2009; 57:334-337.

14. Mahajan SK. Scrub typhus. J Assoc Physicians India 2005; 53:954-958.
15. Kim DM, Kim SW, Choi SH and Yun NR. Clinical and laboratory findings associated with severe scrub typhus. BMC Infect Dis 2010;10:108.

16. Sriwongpan $P$, Krittigamas $P$, Kantipong $P$, Kunyanone $N$, Patumanond $\mathrm{J}$ and Namwongprom $\mathrm{S}$. Clinical indicators for severe prognosis of scrub typhus. Risk Manag Healthc Policy $201311 ; 6: 43-49$

\section{Authors Contribution:}

DM - The treating physician, Concept and Design of the study, analysis and interpretation, manuscript preparation, revision of the manuscript and literature search; BA - The treating physician, analysis and interpretation, manuscript preparation, revision of the manuscript and literature search; CM - The treating physician, Concept and Design of the study, manuscript preparation, revision of the manuscript; SN, SJY, SNY - data collection; PMR - The treating physician, analysis and interpretation, manuscript preparation; SM - Analysis and interpretation, manuscript preparation, revision of the manuscript and literature search. 